\title{
A influência de diferentes formatos de jogos nos aspectos técnicos e táticos de jogadores de futebol
}

http://dx.doi.org/10.11606/1807-5509201900040551

\author{
Eliel Calazans FERREIRA* \\ Felipe Lovaglio BELOZO** \\ Guilherme GRANDIM* \\ Cristian LIZANA* \\ João Claudio MACHADO*** \\ Milton MISUTA* \\ Larissa Rafaela GALATTI* \\ Alcides José SCAGLIA*
}

*Faculdade de Ciências Aplicadas, Universidade Estadual de

Campinas, Limeira, SP, Brasil.

**Universidade Estadual de Campinas, Campinas, SP, Brasil.

${ }_{* * *}$ Universidade Federal do Amazonas, Manaus, AM, Brasil.

\section{Resumo}

0 objetivo do estudo foi investigar a influência da dimensão do campo e do número de jogadores nos aspectos táticos e técnicos em diferentes formatos de jogos. Para tanto, quatorze jogadores de futebol pertencentes à categoria sub-20 participaram de três estruturas de jogos distintas: $\mathrm{Gr}+3 \mathrm{vs} 3+\mathrm{Gr}$ (27x18 m), Gr+6vs6+Gr (52×32 m) e Gr+10vs10+Gr (105×64 m), em duas condições: Jogo Controle e Jogo de Manutenção. Os jogos foram filmados por quatro câmeras de vídeos digitais. Posteriormente, as sequências de imagens foram analisadas no software Dvideo, que possibilitou obter a posição de todos os jogadores em função do tempo e o registro das ações técnicas ocorridas. Desse modo, foram analisadas as variáveis táticas "Centro de Jogo" e "Densidade Ofensiva" (igualdade numérica, superioridade ofensiva e defensiva), assim como as variáveis técnicas de passe, domínio, condução, finalização, desarme, interceptação e carga técnica. Os resultados mostraram diferenças significativas nos indicadores táticos de participação no centro de jogo $(p<0.01)$, igualdade numérica $(p<0.01)$, superioridade defensiva $(p<0.01)$ e ofensiva $(p<0.05)$. Em relação às ações técnicas, foi possivel verificar diferenças significativas no passe $(p<0.01)$, domínio $(p<0.01)$, condução $(p<0.01)$, finalização $(p<0.01)$, desarme $(p<0.05)$, interceptação $(p<0.01)$ e na carga técnica $(p<0.01)$. Concluímos que, a manipulação da dimensão do campo e do número de jogadores influencia a emergência de comportamentos técnico-táticos específicos, o que possibilita treinadores adequarem as estruturas dos jogos para a elaboração de exercícios que estejam de acordo com os seus objetivos pedagógicos.

Palavras-chave: Futebol; Jogos Experimentais; Análise e Desempenho de Tarefas; Educação Física e Treinamento.

\section{Introdução}

Nos últimos anos, o treinamento com jogos (reduzido ou formal) tem sido implementado no futebol, independentemente da idade e do nível dos jogadores ${ }^{1}$. Por respeitar o contexto especifico do jogo, esse método de treinamento permite o desenvolvimento integrado dos aspectos físicos, técnicos e táticos ${ }^{1-3}$. Dessa forma, o treinamento com jogos tem se tornado uma importante ferramenta para o ensino e o treino do futebol ${ }^{4}$.
Diversos estudos procuraram avaliar os aspectos técnicos e, principalmente, os comportamentos táticos em jogos reduzidos ${ }^{1,5-8}$. Nesse sentido, as pesquisas verificaram como a manipulação das referências estruturais e funcionais do jogo ${ }^{9}$ podem influenciar os comportamentos técnicos e táticos, e potencializar o processo de ensino, aprendizagem e treinamento do futebol.

Em relação aos aspectos técnicos, as pesquisas 
têm mostrado que a utilização de diferentes formatos de jogos influencia a relação com a bola dos jogadores. Além disso, um maior número de açōes técnicas foi observado em jogos com menor área por jogador ${ }^{10-12}$.

OWEN et al. ${ }^{8}$ realizaram um estudo com jogadores de futebol profissional e avaliaram a influencia de diferentes formatos de jogos, pequenos (4vs4), médios (5vs5 a 8vs8) e grandes (9vs9 a 11vs11), e constataram diferenças significativas nas ações técnicas de passes realizados, passes recebidos, dribles, cabeceios, desarmes, interceptações e chutes. No entanto, não encontraram diferenças significativas em relação à mudança de direção e bloqueios.

SILVA et al. ${ }^{13}$ avaliaram jogadores brasileiros da categoria sub-15, em três formatos de jogos (3vs3, $4 \mathrm{vs} 4$ e 5vs5), e verificaram diferenças significativas nas ações técnicas de cruzamentos, dribles e chutes no gol. Entretanto, não encontraram diferenças significativas nas açôes técnicas de envolvimento com a bola, passes, passes certos, desarmes e cabeceios.

No que se referem às ações táticas, estudos evidenciaram que a manipulação do tamanho de campo pode influenciar os padrões táticos ${ }^{5,14}$. Desse modo, a estrutura de jogo claramente afeta a quantidade e a qualidade das ações realizadas tanto na fase ofensiva como defensiva ${ }^{6}$.

SiLva et al. ${ }^{6}$ avaliaram o comportamento tático de jogadores de futebol nos formatos de jogos 3vs3 e 6vs6, e observaram que na fase ofensiva os princípios táticos de "Penetração" e "Mobilidade em Profundidade" apresentaram diferenças significativas no $3 \mathrm{vs} 3$ comparado ao 6vs6, enquanto que a "Unidade Ofensiva" ocorreu significativamente mais no $6 \mathrm{vs} 6$ do que no $3 \mathrm{vs} 3 . \mathrm{Na}$ fase defensiva, os princípios táticos de "Contenção" e "Unidade Defensiva" ocorreram mais vezes no 3vs3 do que no 6vs6, enquanto que a "Cobertura Defensiva" e o "Balanço" foram mais freqüentes no 6vs6 comparado ao 3vs3.

O desenvolvimento tecnológico (GPS e videogrametria) permitiu novas abordagens nas análises táticas no futebol baseado em métricas, tais como, método de centróide da equipe, controle do espaço de jogo, rede de passes, participação no centro de jogo, ângulo do passe e densidade ofensiva (superioridade, igualdade e inferioridade numérica $)^{4,7,15,16}$.

Lizana et al. ${ }^{7}$ investigaram dois jogos conceituais reduzidos com diferentes princípios organizacionais (manutenção da posse da bola e progressão ao alvo) no formato de jogo 6vs6. Os autores verificaram que os jogos de manutenção apresentaram diferenças significativas comparado ao jogo de progressão nos indicadores táticos de participação no centro de jogo, igualdade numérica e superioridade defensiva. Esses resultados são de grande importância para compreender como as diferentes regras influenciam o comportamento tático dos jogadores. Nesse sentido, vemos a necessidade de investigar os indicadores táticos em diferentes estruturas de jogos.

Para uma intervenção pedagógica adequada no treinamento com jogos, é necessário compreender as manifestações técnicas e táticas geradas a partir da manipulação de diferentes restrições de tarefas, especialmente dos fatores relacionados com a dimensão do campo e o número de jogadores ${ }^{17}$. Dessa forma, o objetivo do presente estudo consistiu em verificar a influência da dimensão do campo e do número de jogadores nos aspectos técnicos e táticos em diferentes estruturas de jogos. Os resultados deste estudo contribuirão para que os treinadores possam melhor modelar as sessōes de treino de acordo com os objetivos técnicos e táticos.

\section{Método}

\section{Participantes}

Participaram do presente estudo quatorze jogadores de futebol pertencentes à categoria Sub20 (idade: $18 \pm 1.2$ anos) de um clube filiado na Federação Paulista de Futebol, com um histórico de quatro anos de prática na modalidade e nível competitivo estadual. Os treinos eram realizados cinco vezes na semana e durante as sessóes eram introduzidos treino com jogos (reduzido e formal). Os participantes foram confirmados após manifestação por escrito do termo de consentimento livre e esclarecido aprovado pelo Comitê de Ética em pesquisa da Faculdade de Ciências Médicas da Universidade Estadual de Campinas (CAAE: 10855912.0.0000.5404 e parecer no 208.290). 


\section{Abordagem experimental para o problema}

Os jogadores foram submetidos a seis jogos em diferentes dimensóes de campo e número de jogadores. Os jogos, nas estruturas $\mathrm{Gr}+3 \mathrm{vs} 3+\mathrm{Gr}(27 \times 18 \mathrm{~m}), \mathrm{Gr}+6 \mathrm{vs} 6+\mathrm{Gr}(52 \times 32$ $\mathrm{m})$ e $\mathrm{Gr}+10 \mathrm{vs} 10+\mathrm{Gr}(105 \times 64 \mathrm{~m})$ com área por jogador, respectivamente, de $60,75 \mathrm{~m}^{2}, 118,8 \mathrm{~m}^{2}$ e $305 \mathrm{~m}^{2}$, foram realizados em duas condiçôes, sendo que na primeira apresentava somente as regras oficiais do jogo de futebol (Condição 1: jogo controle - JCon) e na segunda foram manipuladas regras com a finalidade de enfatizar o principio tático operacional de manutenção da posse de bola (Condição 2: jogo de manutenção - JMan).

As regras adicionadas foram: 1) cinco passes seguidos no campo ofensivo (um ponto); 2) transitar a bola de um corredor lateral ao outro (um ponto); 3) a equipe que fizesse o gol após cinco passes no campo ofensivo marcava oito pontos; 4) apenas dois toques na bola; 5) quando o jogador descumprisse a regra número quatro, a sua equipe perdia um ponto. Todos os jogos tiveram duração de 30 minutos. Nesse estudo, não foram analisadas as ações dos goleiros. Os jogos foram precedidos por quinze minutos de aquecimento padronizado, sendo realizados no período da tarde sempre no mesmo horário. A ordem dos jogos foi randomizada e o intervalo de descanso entre os JMan de 24 horas.

\section{Procedimentos}

Os jogos foram filmados por quatro câmeras de vídeo digitais (JVC-Everio GZ-HM690 Full HD) com resolução de 1080x1920, padrão NTSC, $30 \mathrm{~Hz}$ de frequência de aquisição. As câmeras foram posicionadas no ponto mais alto da arquibancada, em posição fixa, de forma a enquadrar todo o campo de jogo. Após a filmagem, a sequência de imagens de cada câmera foi transferida para disco rígido do computador (computador Intel ${ }^{\circledR}$ Core $^{\mathrm{TM}}$ i7-2600k, 3.40GHz, memória RAM, 16 GB, placa de vídeo NVIDIA GeForce 9500 GT) e convertidos para o formato AVI (Áudio Vídeo Interleaved) com resolução de 640x480.

O sistema de referência criado está associado ao plano do campo no qual a linha lateral está associada ao eixo $\mathrm{X}$ e a linha de fundo ao eixo Y. As coordenadas reais do sistema de referência foram de uma trena laser (Leica Disto ${ }^{\mathrm{TM}} \mathrm{D} 5$, precisão de $0,001 \mathrm{~m}$ ) e um nivelador laser (Bosch
GPL 5C Professional). O DLT (Direct Linear Transformation) foi utilizado para o processo de calibração e reconstrução bidimensional ${ }^{18}$. O Sistema Dvideo foi utilizado para obter a posição de todos os jogadores em função do tempo e o registro das ações técnicas ocorridas ${ }^{19}$. O rastreamento foi automático e nas situações em que o processo automático não foi possível, houve a intervenção do operador.

\section{Análise tática}

O centro de jogo (CJ) compreende um círculo de raio determinado para cada uma das situações de jogo e centrado na posição da bola (FIGURA 1A). Sendo assim, respeitando o coeficiente de racionalização de densidade dos jogadores proposto por outros estudos ${ }^{20}$, os valores de raio utilizados para o CJ neste estudo foram de cinco metros para a situação de $\mathrm{Gr}+3 \mathrm{vs} 3+\mathrm{Gr}$, sete metros para a situação de $\mathrm{Gr}+6 v s 6+\mathrm{Gr}$ e 915 metros para a situação de Gr+10vs10+Gr. A quantificação relativa à participação no $\mathrm{CJ}(\mathrm{PCJ})$ consiste no número de ocorrências em que cada jogador encontra-se dentro do centro de jogo.

Para cada situação ofensiva foi calculado quantos jogadores, companheiros e adversários, haviam em relação à linha da bola. A linha da bola refere-se a linha paralela à linha de fundo, na qual está associada ao eixo $\mathrm{Y}$ do sistema de referência (FIGURA 1A). Uma vez que a posição da bola e de todos os jogadores foi conhecida, é calculada a quantidade de jogadores posicionados em relação à linha da bola (FIGURA 1B). Sendo assim, a densidade ofensiva, representada pelas relações numéricas e espaciais entre os jogadores de ambas as equipes posicionadas à frente da linha da bola ${ }^{7}$, foi definida em três situações: 1) Igualdade Numérica (IN), isto é, quando a quantidade de jogadores da equipe em fase ofensiva era igual a da equipe em fase defensiva (FIGURA 1B); 2) Superioridade Defensiva (SD), isto é, quando a equipe em fase ofensiva estava em desvantagem numérica em comparação à equipe em fase defensiva (FIGURA 1C); e 3) Superioridade Ofensiva (SO), ou seja, quando a equipe em fase ofensiva estava em vantagem numérica em relação à equipe em fase defensiva (FIGURA 1D). Além disso, foi quantificada a participação no centro de jogo (PCJ) para cada jogador, de maneira que permitisse aceder a importantes informações acerca do envolvimento do mesmo no jogo. 
$r$ raio em metros; linha da bola: corresponde à linha pontilhada associada à posição da bola e paralela ao eixo $Y$ do sistema de referência.
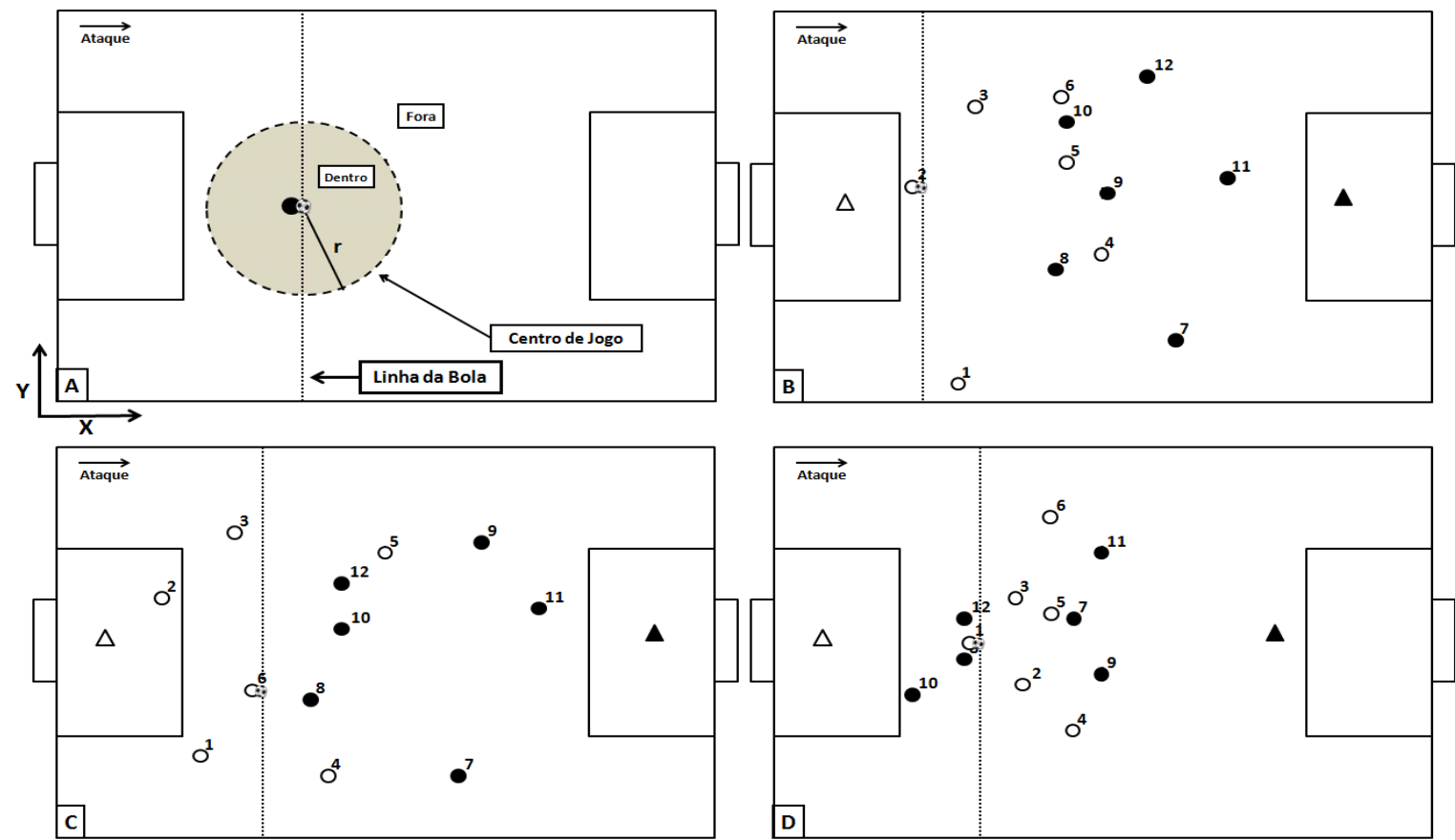

FIGURA 1 -Desenho esquemático dos indicadores táticos: Participação no CJ (A), Igualdade Numérica (B), Superioridade Defensiva (C) e Superioridade Ofensiva (D).

\section{Análise técnica}

As ações técnicas dos jogos foram registradas de acordo com os indicadores utilizados em outro estudo ${ }^{21}$ : passe, domínio, condução, finalização, desarme, interceptação. Para este trabalho, as ações técnicas foram quantificadas e agrupadas considerando o número de ocorrências para cada jogador em cada um dos jogos realizados. Dessa forma, calculou-se uma carga técnica (CT), isto é, a quantidade de ações técnicas realizadas pelo jogador (N) em função do tempo de jogo (T): CT $=\mathrm{NxT}$. A CT se refere ao volume do treino dos aspectos técnicos em contexto de jogo.

\section{Análise dos Dados}

A normalidade dos dados foi verificada com o teste Shapiro-Wilk. A análise dos jogos (ANOVA Oneway) foi realizada tendo como fator o formato do jogo
$(\mathrm{Gr}+3 \mathrm{vs} 3+\mathrm{Gr}, \mathrm{Gr}+6 \mathrm{vs} 6+\mathrm{Gr}$ e Gr+10vs10+Gr) para as duas condições (Condição 1: jogo controle - JCon e Condição 2: jogo de manutenção - JMan). O post hoc de Tukey foi utilizado quando houve um efeito significante considerando os formatos dos jogos. $\mathrm{O}$ teste ANOVA One-way foi aplicado utilizando cada uma das seguintes variáveis: a) aspecto tático: centro de jogo (PCJ); situação de Igualdade Numérica (IN), Superioridade Defensiva (SD) e Superioridade Ofensiva (SO); b) aspecto técnico: passe, domínio, condução, finalização, desarme, interceptação e carga técnica (CT). O nível de significância adotado foi de $p<0.05$. A qualidade dos dados foi confirmada pelo teste de fiabilidade (correlação intra-observador). Sendo assim, o mesmo observador analisou um jogo por duas oportunidades, num intervalo de 15 dias. Os valores de coeficiente intra-classe variaram de 0.95 a 0.99 , considerando todas as variáveis relativas a aspectos táticos e técnicos.

\section{Resultados}

\section{Análise tática}

Nas duas condições de jogos, a ANOVA One-way mostrou diferenças significativas nos indicadores táticos de PCJ (JCon: $\mathrm{p}<0.01$, JMan: $\mathrm{p}<0.01$ ), IN (JCon: $\mathrm{p}<0.01$, JMan: $\mathrm{p}<0.01$ ), SD (JCon: $\mathrm{p}<0.01$, JMan: $\mathrm{p}<0.01)$ e SO (JCon: $\mathrm{p}<0.01$, JMan: $\mathrm{p}<0.01)$. A comparação do post hoc de Tukey dos indicadores táticos entre os formatos de jogos,da mesma condição, são 
indicados na FIGURA 2A e 2B.

$\mathrm{Na}$ comparação entre os jogos controle e manutenção, o $\mathrm{Gr}+3 \mathrm{vs} 3+\mathrm{Gr}$ e o $\mathrm{Gr}+6 \mathrm{vs} 6+\mathrm{Gr}$ apresentaram diferenças significativas nos indicadores táticos de PCJ (Gr+3vs3+Gr: $\mathrm{p}<0.01$, Gr+6vs6+Gr: $p<0.01), I N(G r+3 v s 3+G r: p<0.05$, $\mathrm{Gr}+6 \mathrm{vs} 6+\mathrm{Gr}: \mathrm{p}<0.01)$ e $\mathrm{SO}(\mathrm{Gr}+3 \mathrm{vs} 3+\mathrm{Gr}$ : $\mathrm{p}<0.01, \mathrm{Gr}+6 \mathrm{vs} 6+\mathrm{Gr}: \mathrm{p}<0.01)$. Não foram observadas diferenças significativas na SD
$(\mathrm{Gr}+3 \mathrm{vs3}+\mathrm{Gr}: \mathrm{p}>0.05, \mathrm{Gr}+6 \mathrm{vs} 6+\mathrm{Gr}: \mathrm{p}>0.05)$

(FIGURA 2A e 2B).

$\mathrm{O} \mathrm{Gr}+10 \mathrm{vs} 10+\mathrm{Gr}$ apresentou diferenças significativas no indicador tático de PCJ $(\mathrm{Gr}+10 \mathrm{v}$ 10+Gr: p<0.01). Não foram verificadas diferenças significativas nos indicadores táticos de IN $(\mathrm{Gr}+10 \mathrm{vs} 10+\mathrm{Gr}$ : p>0.05), SD $(\mathrm{Gr}+10 \mathrm{vs} 10+\mathrm{Gr}: \mathrm{p}>0.05)$ e SO (Gr+10vs10+Gr: p>0.05) (FIGURA 2A e 2B).
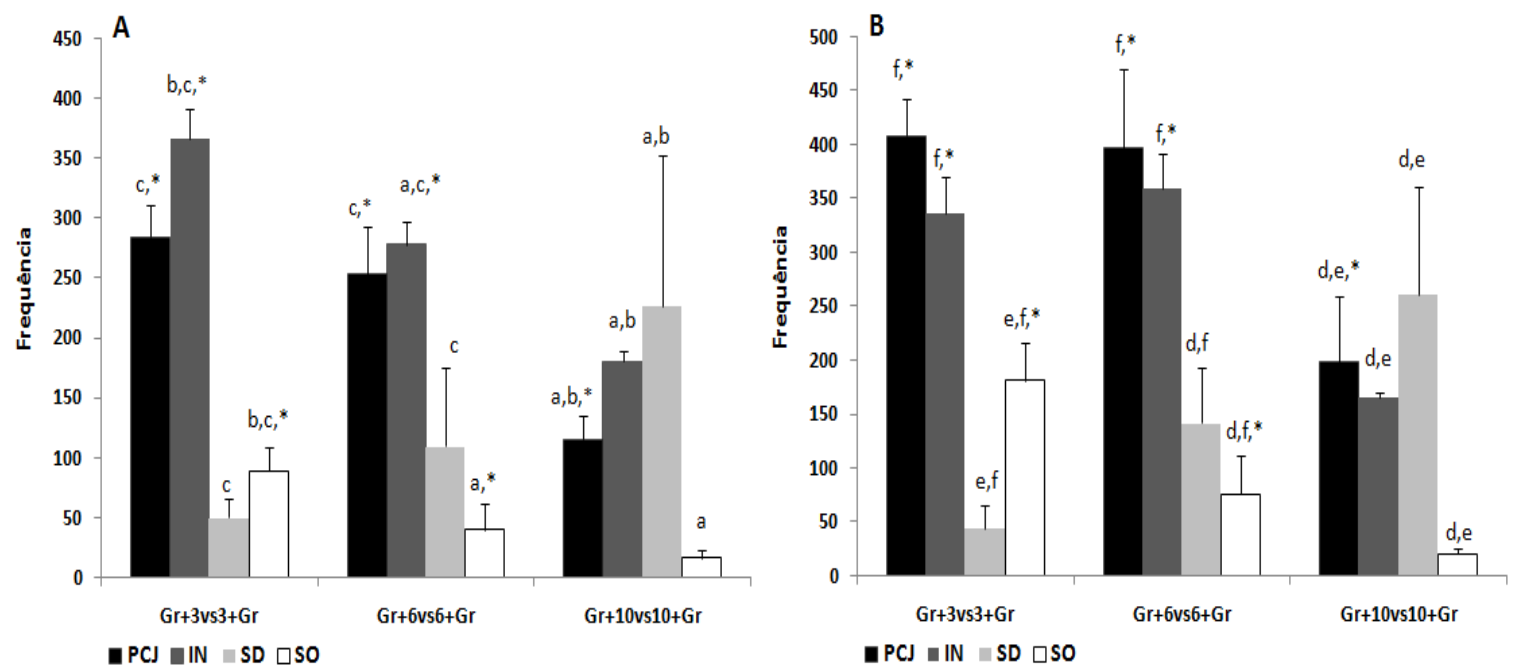

Diferenças significativas entre os formatos de JCon : $: \mathrm{Gr}+3 v s 3+\mathrm{Gr}$, ${ }^{b} \mathrm{Gr}+6 \mathrm{vs} 6+\mathrm{Gr} \quad \mathrm{e}$ ${ }^{\circ} \mathrm{Gr}+10 \mathrm{vs} 10+\mathrm{Gr}$. Diferenças significativas entre os formatos de JMan : ${ }^{d} \mathrm{Gr}+3 \mathrm{vs} 3+\mathrm{Gr}$, ${ }^{e} G r+6 v s 6+G r \quad e$ $\mathrm{G} r+10 \mathrm{vs} 10+\mathrm{Gr}$

*Diferenças significativas entre as condições de jogo controle e manutenção.

Nível de significância: $p<0,05$.

FIGURA 2 -Média e desvio padrão dos indicadores táticos: Participação no CJ (PCJ), Igualdade Numérica (IN), Superioridade Defensiva (SD) e Superioridade Ofensiva (SO) nos formatos Gr+3vs3+Gr, Gr+6vs6+Gr e Gr+10vs10+Gr em jogos controle (A) e manutenção (B).

\section{Análise técnica}

A ANOVA One-way mostrou diferenças significativas nas ações técnicas de passe (JCon: $\mathrm{p}<0.05$, JMan: $\mathrm{p}<0.01$ ), domínio (JCon: $\mathrm{p}<0.01$, JMan: $\mathrm{p}<0.01$ ), condução (JCon: $\mathrm{p}<0.01$ ), finalização (JCon: $\mathrm{p}<0.01$ ), desarme (JCon: $\mathrm{p}<0.01)$ e interceptação (JCon: $\mathrm{p}<0.01$, JMan: $\mathrm{p}<0.01)$. Não foram verificadas diferenças significativas nas ações técnicas de condução (JMan: p>0.05), finalização (JMan: p>0.05) e desarme (JMan: p>0.05). A comparação do post hoc de Tukey dos indicadores táticos entre os formatos de jogos, da mesma condição, são indicados na TABELA 1.

$\mathrm{Na}$ comparação entre os jogos controle e manutenção, o $\mathrm{Gr}+3 \mathrm{vs} 3+\mathrm{Gr}$ apresentou diferenças significativas nas ações técnicas de passe $(\mathrm{p}<0.01)$, domínio $(\mathrm{p}<0.01)$, condução $(p<0.01)$, finalização $(p<0.01)$ e interceptação $(\mathrm{p}<0.01)$. Não foram observadas diferenças significativas na ação técnica de desarme $(\mathrm{p}>0.05)$ (TABELA 1)

$\mathrm{O} \mathrm{Gr}+6 \mathrm{vs} 6+\mathrm{Gr}$ apresentou diferenças significativas nas ações técnicas de passe $(\mathrm{p}<0.01)$, condução $(\mathrm{p}<0.01)$ e finalização $(\mathrm{p}<0.01)$. Não foram observadas diferenças significativas nas açôes técnicas de domínio ( $\mathrm{p}>0.05)$, desarme $(p>0.05)$ e interceptação $(p>0.05)$ (TABELA 1$)$.

$\mathrm{O} \mathrm{Gr}+10 \mathrm{vs} 10+\mathrm{Gr}$ apresentou diferenças significativas na ação técnica de condução $(\mathrm{p}<0.05)$. Não foram observadas diferenças significativas nas ações técnicas de passe ( $p>0.05)$, domínio ( $p>0.05)$, finalização $(p>0.05)$, desarme $(\mathrm{p}>0.05)$ e interceptação $(\mathrm{p}>0.05)$ (TABELA 1$)$.

Os resultados da ANOVA Oneway, nas duas condições de jogos, mostraram diferenças significativas na CT (JCon: $\mathrm{p}<0.01$, JMan: $\mathrm{p}<0.01)$. A comparação do post hoc de Tukey dos indicadores táticos entre os formatos de jogos são indicados na FIGURA 3. Não foram encontradas diferenças significativas entre as condições de jogos na CT $(\mathrm{Gr}+3 \mathrm{vs} 3+\mathrm{Gr}$ : p >0.05, Gr+6vs6+Gr: $\mathrm{p}>0.05, \mathrm{Gr}+10 \mathrm{vs} 10+\mathrm{Gr}: \mathrm{p}>0.05)$ (FIGURA 3). 
Diferenças significativas em relação aos JCon: ${ }^{\mathrm{a}} \mathrm{Gr}+3 \mathrm{vs} 3+\mathrm{Gr}$, ${ }^{b} \mathrm{Gr}+6 \mathrm{vs} 6+\mathrm{Gr} \quad \mathrm{e}$ ${ }^{\circ} \mathrm{Gr}+10 \mathrm{vs} 10+\mathrm{Gr}$.

Diferenças significativas em relação aos JMan: ${ }^{\mathrm{d}} \mathrm{Gr}+3 \mathrm{vs} 3+\mathrm{Gr}$, ${ }^{e} \mathrm{Gr}+6 \mathrm{vs} 6+\mathrm{Gr} \quad \mathrm{e}$ ${ }^{\prime} \mathrm{Gr}+10 \mathrm{vs} 10+\mathrm{Gr}$. ${ }^{*}$ Diferenças significativas entre as condições de jogos controle e manutenção.

Nível de significância: $p<0.05$.

Diferenças significativas entre os formatos de jogos: * $\mathrm{Gr}+3 \mathrm{vs} 3+\mathrm{Gr}$, ${ }^{*} \mathrm{Gr}+6 \mathrm{vs} 6+\mathrm{Gr} \quad \mathrm{e}$ ${ }^{\$} \mathrm{Gr}+10 v$ s10+Gr.

Nível de significância: $p<0,05$.

TABELA 1 -Média e desvio padrão das ações técnicas nas diferentes estruturas e condições de jogo.

\begin{tabular}{lcccccc}
\hline & \multicolumn{3}{c}{ JCon } & \multicolumn{3}{c}{ JMan } \\
\hline Ações técnicas & $\mathrm{Gr}+3 \mathrm{vs} 3+\mathrm{Gr}$ & $\mathrm{Gr}+6 \mathrm{vs} 6+\mathrm{Gr}$ & $\mathrm{Gr}+10 \mathrm{vs} 10+\mathrm{Gr}$ & $\mathrm{Gr}+3 \mathrm{vs} 3+\mathrm{Gr}$ & $\mathrm{Gr}+6 \mathrm{vs} 6+\mathrm{Gr}$ & $\mathrm{Gr}+10 \mathrm{vs} 10+\mathrm{Gr}$ \\
\hline Passe & $29,8 \pm 8,1^{\mathrm{c} *}$ & $24,8 \pm 8,6^{*}$ & $18,8 \pm 10,4^{\mathrm{a}}$ & $69,9 \pm 11,4^{\mathrm{e}, \mathrm{f} *}$ & $48,1 \pm 11,4^{\mathrm{d}, \mathrm{f} *}$ & $28,9 \pm 7,1^{\mathrm{d}, \mathrm{e}}$ \\
Domínio & $36,4 \pm 8,2^{\mathrm{b}, \mathrm{c} *}$ & $22,9 \pm 8,4^{\mathrm{a}}$ & $15,1 \pm 9,5^{\mathrm{a}}$ & $48,0 \pm 9,2^{\mathrm{e}, \mathrm{f} *}$ & $29,4 \pm 10,5^{\mathrm{d}, \mathrm{f}}$ & $18,4 \pm 6,1^{\mathrm{d}, \mathrm{e}}$ \\
Condução & $20,6 \pm 8,1^{\mathrm{b}, c^{*}}$ & $11,9 \pm 5,3^{\mathrm{a} *}$ & $7,9 \pm 5,3^{\mathrm{a} *}$ & $0,3 \pm 0,6^{*}$ & $0,0 \pm 0,0^{*}$ & $0,0 \pm 0,0^{*}$ \\
Finalização & $14,9 \pm 4,9^{\mathrm{b}, * *}$ & $4,1 \pm 2,8^{\mathrm{a}, *}$ & $0,3 \pm 0,6^{\mathrm{a}, \mathrm{b}}$ & $0,7 \pm 1,1^{*}$ & $0,6 \pm 0,8^{*}$ & $0,0 \pm 0,0$ \\
Desarme & $5,1 \pm 2,7^{\mathrm{c}}$ & $3,4 \pm 1,9$ & $0,9 \pm 1,1^{\mathrm{a}}$ & $3,3 \pm 1,8$ & $2,5 \pm 1,9$ & $1,3 \pm 1,3$ \\
Interceptação & $6,5 \pm 3,3^{* *}$ & $3,9 \pm 2,7$ & $2,5 \pm 1,8^{\mathrm{a}}$ & $12,5 \pm 3,8^{\mathrm{e}, \mathrm{f} *}$ & $6,9 \pm 2,9^{\mathrm{d}, \mathrm{f}}$ & $3,6 \pm 2,3^{\mathrm{d}, \mathrm{e}}$ \\
\hline
\end{tabular}

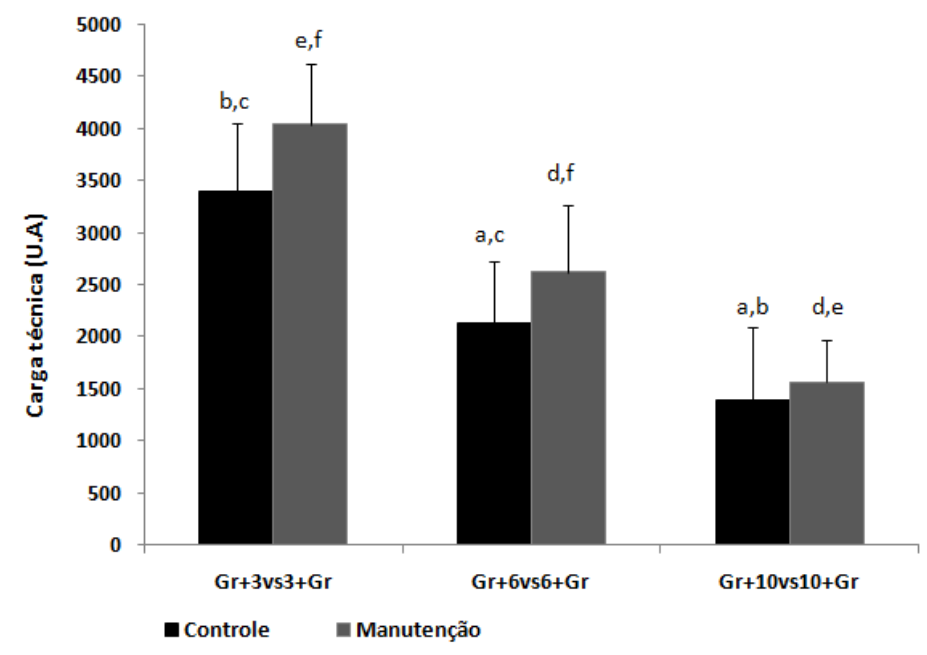

FIGURA 3 -Média e desvio padrão da CT nas diferentes estruturas e condições de jogo.

\section{Discussão}

O presente estudo teve como objetivo verificar a influência da dimensão do campo e do número de jogadores nos aspectos táticos e técnicos em diferentes estruturas de jogo. Os resultados mostraram que os indicadores táticos, o desempenho técnico e a CT apresentaram diferenças significativas entre as estruturas de jogos $\mathrm{Gr}+3 \mathrm{vs} 3+\mathrm{Gr}, \mathrm{Gr}+6 \mathrm{vs} 6+\mathrm{Gr}$ e $\mathrm{Gr}+10 \mathrm{vs} 10+\mathrm{Gr}$.

Nas duas condições de jogos, os resultados mostraram que quanto maior a dimensão do campo e número de jogadores menor é a PCJ. Sendo assim, pode-se inferir que em estruturas menores, o jogo fica mais concentrado, fazendo com que os jogadores participem mais ativamente dentro do CJ, conseqüentemente, o portador da bola sofre uma maior e constante pressão. No estudo, também foi possível constatar que, em estruturas menores de jogo, os jogadores realizaram maior quantidade de ações técnicas, e como se trataram de ações em que os jogadores estiveram em contato com a bola e/ou próximo do portador da mesma, é possível, portanto, destacar a maior participação dos jogadores no centro de jogo. No entanto, nos formatos maiores, a pressão no CJ é exercida em regiōes especificas do campo de jogo, fazendo os jogadores participarem menos do CJ. É importante destacar que o CJ é a principal referência espacial para se cumprir os princípios táticos fundamentais do jogo de futebol, em que os jogadores posicionados dentro, próximo ou distante do CJ podem influenciar nas açóes do portador da bola contribuindo para o desempenho tático da equipe ${ }^{6,20}$.

Em outras investigações ${ }^{6}$, que procuraram comparar o comportamento tático em jogos nos formatos $\mathrm{Gr}+3 \mathrm{vs} 3+\mathrm{Gr}$ e $\mathrm{Gr}+6 \mathrm{vs} 6+\mathrm{Gr}$, foi possível constatar que no $\mathrm{Gr}+3 \mathrm{vs} 3+\mathrm{Gr}$ os jogadores desempenharam mais ações táticas ofensivas de penetração, mobilidade e uma quantidade 
menor de ações de unidade ofensiva, enquanto que defensivamente uma maior quantidade de contenção e unidade defensiva. Esses resultados, em conjunto com os observados neste estudo, sugerem que, em função dos objetivos pretendidos, o treinador deve considerar a dimensão do campo e o número de jogadores no momento de planejar os treinamentos com jogos.

Nos JCon, quando se aumenta a dimensão do campo e o número de jogadores ocorre uma redução significativa nas situaçôes de IN. No entanto, nos JMan, nota-se um aumento, não significativo, no $\mathrm{Gr}+6 v s 6+\mathrm{Gr}$ em comparação ao Gr+3vs3+Gr. Este aumento pode estar relacionado com a inclusão das regras nos JMan, que induz uma quantidade maior de situações de equilíbrio numérico. Pode-se considerar as situações de IN como um limiar entre a $\mathrm{SO}$ e SD. A equipe em fase ofensiva, que explora as situações de IN, pode evoluir, rapidamente, para SO e desequilibrar momentaneamente a organização defensiva adversária. Contrariamente, a equipe em fase defensiva pode evoluir para uma situação de superioridade numérica e oferecer uma maior segurança ao sistema defensivo. Dessa forma, os resultados mostraram que nos jogos $\mathrm{Gr}+3 \mathrm{vs} 3+\mathrm{Gr}$ (JCon e JMan), as situaçôes de IN tendem a evoluir mais para situações de $\mathrm{SO}$, enquanto que no Gr+6vs6+Gr e Gr+10vs10+Gr para situações de SD.

Nas duas condições de jogo, o aumento na dimensão do campo e número de jogadores proporcionou o surgimento de situaçôes de SD. Outros estudos ${ }^{22,23}$ destacaram a importância da realização dos princípios táticos gerais (i.e. criar superioridade numérica, evitar a igualdade numérica e não permitir a inferioridade numérica), que possuem esta nomenclatura por serem comuns às fases do jogo e aos demais princípios de jogo. Isso porque, ao criar um contexto de superioridade numérica no CJ, a equipe aumenta as chances de manter a posse de bola, progredir pelo espaço de jogo e criar situações de finalização (fase ofensiva) ou pressionar a equipe adversária e recuperar a possa da bola (fase defensiva) ${ }^{22,24}$. Dessa forma, os resultados demonstraram que os jogos com menor estrutura ofereceram mais oportunidade de treinar os comportamentos táticos ofensivos em vantagem numérica, uma vez que em jogos maiores, os jogadores, em fase defensiva, apresentaram forte tendência em se posicionar atrás da linha da bola.

Neste estudo, os valores da CT na estrutura $\mathrm{Gr}+3 \mathrm{vs} 3+\mathrm{Gr}$ apresentaram diferenças significativamente superiores em relação às demais estruturas, nas duas condições de jogo. Sendo assim, observa-se uma relação inversamente proporcional, ou seja, quando se diminui a dimensão do campo e o número de jogadores ocorre o aumento na frequência das ações técnicas. Tais resultados corroboraram com os achados de outras investigaçôes ${ }^{5,8}$, em que também foi possível observar o maior número de açôes técnicas de passe, domínio, dribles, cabeceio, desarme e finalização realizadas em jogos com dimensões reduzidas e número menor de jogadores.

Nos JCon foram observadas diferenças significativas nas estruturas $\mathrm{Gr}+3 \mathrm{vs} 3+\mathrm{Gr}$ e Gr+10vs10+Gr nas ações de passe, enquanto que nos JMan observaram-se diferenças significativas em todas as estruturas. Entre as condiçôes de jogo, foi possível constatar diferenças significativas nos passes realizados entre as estruturas $\mathrm{Gr}+3 \mathrm{vs} 3+\mathrm{Gr}$ e $\mathrm{Gr}+6 v s 6+\mathrm{Gr}$. O aumento dos passes se justifica pela diminuição na estrutura de jogo (campo reduzido com poucos jogadores), consequentemente, os jogadores exercem maior pressão no CJ. Portanto, a tomada de decisão do portador da bola é mais rápida, aumentando o ritmo de passes ${ }^{8}$.

Neste estudo, foi observada uma diminuição na frequência das ações de domínio com o aumento da dimensão do campo e do número de jogadores, em ambas às condiçôes de jogos. O domínio é o segundo fundamento mais realizado em uma partida de futebol e mantém uma relação quantitativa com as ações de passe. Dessa forma, as possíveis diferenças encontradas, entre os dois fundamentos, podem estar relacionadas com a execução do passe ao obter o primeiro toque na bola. No entanto, ao optar pela preferência de passar a bola no primeiro toque, pode-se aumentar a frequência de erros nas açôes de passe ${ }^{25}$. Sendo assim, exercer a ação de domínio permite ao jogador melhor controle da bola antes de realizar o passe, aumentando as chances de acerto. Portanto, nos formatos de jogos com estruturas menores de jogo, realizar as açôes de domínio foi mais difícil devido ao fato da constante pressão dos adversários.

O JCon, no formato Gr+3vs3+Gr, apresentou diferenças significativamente superiores em relação as demais estruturas nas ações de condução e finalização, corroborando com outros estudos ${ }^{5,8,26}$. Nas ações de condução, os resultados sugerem que nos jogos com estruturas menores existem poucas opções para se passar a bola, forçando os jogadores a desempenhar mais ações de condução para criar espaços no campo ${ }^{8}$. Em relação às ações de finalização, o maior índice encontrado nos 
jogos de menor estrutura pode estar relacionado à proximidade com que os jogadores estão em relação à baliza adversária, oferecendo mais oportunidades para rematar e marcar gols ${ }^{5,26}$. Sendo assim, jogos com menor estrutura proporcionaram maiores oportunidades aos jogadores de realizar ações de ataque e defesa ${ }^{8}$.

Quanto às ações de desarme e interceptação, foi possível observar que em estruturas menores de jogo maior é a quantidade dessas ações realizadas pelos jogadores. Os resultados sugerem que as ações de desarme foram estimuladas pelas ações de condução de bola, uma vez que na estrutura $\mathrm{Gr}+3 \mathrm{vs} 3+\mathrm{Gr}$ ocorre maior quantidade de situações de 1vs1. Por sua vez, as ações de interceptações estão relacionadas com os elevados volumes de passes que são mais estimulados nos jogos com menor estrutura.

Os resultados do estudo sugerem aos treinadores que a manipulação da dimensão do campo e do número de jogadores pode ser utilizada como estratégia pedagógica para gerar comportamentos técnicos e táticos específicos, de acordo com os objetivos da sessão de treino. Dessa forma, os treinadores podem utilizar estruturas menores de jogo para proporcionar maior CT, já que há maior quantidade de ações técnicas realizadas, uma maior PCJ, constatando que os jogadores participam mais do jogo, independentemente das condições. Além disso, essas estruturas proporcionam situações de SO, favorecendo as tomadas de decisões em situações ofensivas com maior número de jogadores. Contrariamente, as estruturas maiores de jogos podem ser utilizadas para enfatizar situações de SD e de menor CT. Portanto, recomenda-se uma distribuição equilibrada dos vários formatos de jogos no planejamento dos microciclos semanais para favorecer adaptações especificas próximas da realidade da competição formal.

\section{Abstract}

The influence of different game formats in the technical and tactical aspects of soccer players

The aim of the study was to investigate the influence of field size and number of players on the technical and tactical aspects in different game formats. For this purpose, fourteen soccer players in U20 category participated in three different game formats: Gk+3vs3+Gk (27x18 m), Gk+6vs6+Gk (52×32 m) and Gk+10vs10+Gk (105x64 m), in two conditions: Control Game - CG and Maintenance Game - MG. The games were filmed by four digital video cameras. Subsequently, the image sequences were analyzed in Dvideo software that allowed to obtain the position of all the players in function of time and the record of technical actions occurred. Thus, we analyzed the tactics variables "Game center" and "Offensive density" (numerical equality, offensive and defensive superiority), as well as technical variables (pass, control, driving, finalization, tackling, interception) and technical load. The results showed significant differences in the tactical indicators of participation in the game center $(p<0.01)$, numerical equality $(p<0.01)$, defense superiority $(p<0.01)$ and offensive $(p<0.05)$. In relation to the technical actions, it was possible to verify significant differences in the pass $(p<0.01)$, control $(p<0.01)$, driving $(p<0.01)$, finalization $(p<0.01)$, tackling $(p<0.01)$, interception $(p<0.01)$ and technical load $(p<0.01)$. We conclude that the manipulations of field dimension and the player number influence thespecifics technical-tactical behaviours, which enables coaches fit the game structures for the development of task according to your pedagogical goals.

KEYwoRds: Soccer; Experimental Games; Task Performance and Analysis; Physical Education and Training.

\section{References}

1. Fradua L, Zubillaga A, Caro O, Iván Fernández-García A, Ruiz-Ruiz C, Tenga A. Designing small-sided games for training tactical aspects in soccer: extrapolating pitch sizes from full-size professional matches. J Sports Sci. 2013;31(6):573-81.

2. Hill-Haas SV, Rowsell GJ, Dawson BTC, Coutts AJ. Acute physiological responses and time-motion characteristics 
of two small-sided training regimes in youth soccer players. J Strength Cond Res. 2009;23(1):111-5.

3. Rampinini E, Impellizzeri FM, Castagna C, Abt G, Chamari K, Sassi AM, Marcora SM. Factors influencing physiological responses to small-sided soccer games. J Sports Sci. 2007;25(6):659-66.

4. Folgado H, Lemmink KA, Frencken W, Sampaio J. Length, width and centroid distance as measures of teams tactical performance in youth football. Eur J Sport Sci. 2014;14(Suppl1):S487-92.

5. Almeida $\mathrm{CH}$, Ferreira AP, Volossovitch A. Offensive sequences in youth soccer: effects of experience and small-sided games. J Hum Kinet. 2013;36:97-106.

6. Silva B, Garganta J, Santos R, Teoldo I. Comparing Tactical Behaviour of Soccer Players in 3 vs. 3 and 6 vs. 6 SmallSided Games. J Hum Kinet. 2014;41:191-202.

7. Lizana CJR, Reverdito RS, Brenzikofer R, Macedo DV, Misuta MS, Scaglia AJ. Technical and tactical soccer players' performance in conceptual small-sided games. Motriz: J Phys Ed. 2015;21(3):312-20.

8. Owen AL, Wong DP, Paul D, Dellal A. Physical and Technical Comparisons between Various-Sided Games within Professional Soccer. Int J Sports Med. 2014;35(4):286-92.

9. Scaglia AJ, Reverdito R, Leonardo L, Lizana C. O ensino dos jogos esportivos coletivos: as competências essenciais e a lógica do jogo em meio ao processo de organizacional sistêmico. Movimento (ESEF/UFRGS). 2013;19(4):227-49.

10. Casamichana D, Castellano J. Time-motion, heart rate, perceptual and motor behaviour demands in small-sides soccer games: effects of pitch size. J Sports Sci. 2010;28(14):1615-23.

11. Owen AL, Wong DP, McKenna M, Dellal A. Heart rate responses and technical comparison between small-vs. largesided games in elite professional soccer. The Journal of Strength \& Conditioning Research. 2011;25(8):2104-10.

12. Kelly DM, Drust B. The effect of pitch dimensions on heart rate responses and technical demands of small-sided soccer games in elite players. J Sci Med Sport. 2009;12(4):475-9.

13. da Silva CD, Impellizzeri FM, Natali AJ, de Lima JR, Bara-Filho MG, Silami-Garçia E, et al. Exercise intensity and technical demands of small-sided games in young Brazilian soccer players: effect of number of players, maturation, and reliability. J Strength Cond Res. 2011;25(10):2746-51.

14. Costa IT, Garganta J, Greco PJ, Mesquita I, Seabra A. Influence of Relative Age Effects and Quality of Tactical Behaviour in the Performance of Youth Soccer Players. Int J Perf Anal Spor. 2010;10(2):82-97.

15. Moura FA, Martins LEB, Anido RO, Ruffino PRC, Barros RM, Cunha SA. A spectral analysis of team dynamics and tactics in Brazilian football. J sports sci. 2013;31(14):1568-77.

16. Gama J, Passos P, Davids K, Relvas H, Ribeiro J, Vaz V, et al. Network analysis and intra-team activity in attacking phases of professional football. Int J Perf Anal Spor. 2014;14(3):692-708.

17. Aguiar M, Botelho G, Lago C, Maças V, Sampaio J. A Review on the Effects of Soccer Small-Sided Games. J Hum Kinet. 2012;33:103-113.

18. Abdel-Aziz YI, Karara HM. Direct linear transformation from comparator coordinates into object-space coordinates. Synposium Onclosee-Rane Photogrammetrry. Urbana: ASP/UI; 1971. p.1-18.

19. Figueroa PJ, Leite NJ, Barros RM. A flexible software for tracking of markers used in human motion analysis. Comput Methods Programs Biomed. 2003;72(2):155-65.

20. Costa IT, Garganta J, Greco PJ, Mesquita I, Maia J. Sistema de avaliação táctica no Futebol (FUT-SAT): Desenvolvimento e validação preliminar. Motri. 2011;7(1):69-84.

21. Añon IC, Lizana C, Calazans E, Machado J, da Costa I. Performance da equipe do Barcelona e seus adversários nos jogos finais da Champions League e da Copa do Mundo de Clubes FIFA 2010. Rev andal med del deporte. 2014;7(1):1320.

22. Costa IT, Garganta J, Greco PJ, Mesquita I. Princípios táticos do jogo de futebol: Conceitos e aplicação. Motriz: J Phys Ed. 2009;15(3):657-68.

23. Garganta J, Pinto J. O ensino do futebol. In: Graça A, Oliveira J, editors. O ensino dos Jogos Desportivos. Porto: CEJD/FCDEF-UP; 1994. p.97-137. 
Ferreira EC, et al.

24. Bayer C. O ensino dos desportos colectivos. Lisboa: Dinalivros; 1994.252 p.

25. Dellal A, Lago-Penas C, Wong dP, Chamari K. Effect of the number of ball contacts within bouts of 4 vs. 4 small-sided soccer games. Int J Sports Physiol Perform. 2011;6(3):322-33.

26. Katis A, Kellis E. Effects of small-sided games on physical conditioning and performance in young soccer players. J Sports Med. 2009;8(3):374-80.

ENDEREÇO DE CORRESPONDÊNCIA:

Eliel Calazans Ferreira

Rua Pedro Zaccaria, 1300 - Jd. Santa Luiza

Limeira - SP - BRASIL

CEP: 13484350

E-mail: elielcalazans@gmail.com
Submetido: 16/08/2016

$1^{\text {a }}$ revisão: $31 / 10 / 2016$

$2^{\text {a }}$ revisão: $20 / 01 / 2017$

Aceito: 09/03/2017 